\title{
Application of $\beta$-glucosidase Immobilized on Chitosan microspheres in Degradation of Polydatin in Polygonum cuspidatum
}

\author{
Wei Zong,"\#, Shan Liü,\#, Jeonyun Yun ${ }^{3, \#}$, Xiong Xiao ${ }^{3, \#}$, Zujun Deng ${ }^{1,2}, \mathrm{He} \mathrm{Li}^{1,2, *}$ \\ ${ }^{1}$ School of Life Sciences and Biopharmaceuticals, Guangdong Pharmaceutical University, Guangzhou, 511400, China \\ ${ }^{2}$ Guangdong Key Laboratory of Bioactive Drug Research, Guangzhou, 511400, China; \\ ${ }^{3}$ Guangzhou Yachun Cosmetics Manufacturing Co., Ltd, Guangzhou, 510080, China \\ \#these authors contributed equally to this work
}

\begin{abstract}
Resveratrol in Polygonum cuspidatum is a $\beta$-glycoside, which can be hydrolyzed to resveratrol by $\beta$-glucosidase. it is an efficient production process to degrade polydatin from Polygonum cuspidatum extract by immobilized $\beta$-glucosidase. It is of great significance to explore suitable immobilization conditions to improve the catalytic efficiency and reusability of $\beta$-glucosidase for polydatin degradation and cost reduction. In this paper, the recombinant Escherichia coli bgl2238, which was screened and constructed from corn soil of Heilongjiang Province in the early laboratory, was immobilized by chitosan adsorption and glutaraldehyde crosslinking. The preparation conditions and immobilization process of bgl2238 were determined by single factor method: the optimal crosslinking time was $1 \mathrm{~h}$, the optimal crosslinking temperature was $20{ }^{\circ} \mathrm{C}$, the recovery rate of enzyme activity of bgl2238 was $87 \%$, and the enzyme activity was $859.65 \mathrm{mU} / \mathrm{g}$. The optimum temperature of the immobilized bgl2238 is $50^{\circ} \mathrm{C}$, which is $6^{\circ} \mathrm{C}$ higher than that of the free bgl2238, and the temperature stability and $\mathrm{pH}$ stability are improved. After six consecutive hydrolysis of Polygonum cuspidatum, the degradation rate of polydatin is still over $70 \%$, which proves that the immobilized bgl2238 has good reusability. This will be helpful to evaluate the application prospect of $\beta$ - glucosidase immobilized in this system and determine the best conditions for its production.
\end{abstract}

\section{Introduction}

Resveratrol is a kind of natural antioxidant polyphenol. Various studies have shown that resveratrol has the characteristics of good health, can prevent or slow down the process of various pathological conditions, and can treat cancer and various disease[1].In clinical trials, resveratrol has therapeutic effects on cancer[2-6], obesity[7-9], diabetes[10,11], neurological[12-14] and cardiovascular diseases[15-17], and has significant antibacterial activities against a variety of bacteria, viruses and fungi[18, 19]. In recent years, resveratrol has been widely concerned because of its various health benefits. Resveratrol is widely found in many kinds of edible plants, such as Polygonum cuspidatum, grape and peanut[20]. Polygonum cuspidatum is a kind of medicinal plant. In addition to resveratrol, Polygonum cuspidatum also contains high content of polydatin. polydatin is a $\beta$ glycoside, which can be hydrolyzed to resveratrol by $\beta$ glucosidase. Therefore, Polygonum cuspidatum is the best raw material for the production of resveratrol in industry $[21,22]$.

B-glucosidase can catalyze the hydrolysis of $\beta$-Dglucosidase bond and convert polyglycoside into resveratrol, but the low yield of $\beta$-glucosidase limits its use in industry. Through the experimental design of[2] recombinant $\beta$-glucosidase, the yield can be further improved [23, 24]. Compared with free enzyme, immobilized enzyme has become a research hotspot because of its high stability, reusability, easy separation of products after catalytic reaction and low industrial cost. Immobilized bg12238 can not only avoid the need of separation and purification, but also avoid the need of separation and purification in use It can make bgl2238 reused, improve the stability of temperature and $\mathrm{pH}$, and reduce the industrial cost of degradation of Polygonum cuspidatum to a large extent[25].

Various carriers and methods have been used to immobilize the enzyme. The selection of immobilization methods and appropriate carriers are very important for maintaining the activity and stability of the enzyme [2628]. Chitosan is one of the most widely used carrier materials for enzyme immobilization at present. Chitosan has excellent properties such as non-toxic, biocompatibility and plasticity, and it can chelate metal ions. The thermal stability and enzyme activity of the enzyme immobilized by chitosan have been greatly improved [29]. However, the binding force of chitosan and enzyme is weak, so it is often used to fix the enzyme together with the crosslinker glutaraldehyde, which can further generate the adsorption bond to stabilize the 
interaction between the enzyme and the carrier, and improve the stability of the fixed enzyme [30]. The purpose of this study was to obtain the optimal conditions for degradation of polyglycosides by immobilized $\beta$ glucosidase. The effects of glutaraldehyde concentration, $\mathrm{pH}$, time and temperature on the enzyme activity were studied.

\section{Materials and Methods}

\subsection{Chemicals and Materials}

4-nitrophenyl- $\beta$-d-glucopyranoside (pNPG) and pNP were purchased from Shanghai biotechnology company. The recombinant $\beta$ - glucosidase crude enzyme solution (bg12238) was preserved in our laboratory. High density chitosan (degree of deacetylation is $85 \%$ - $95 \%$ ) was purchased from onco, $50 \%$ glutaraldehyde was purchased from Aladdin biochemical company. Polygonum cuspidatum powder was obtained from Chinese herbal pieces. Resveratrol standard and resveratrol standard were purchased from Chinese herbal pieces Dalian Meilun biological Co., Ltd.; methanol is the first-class chromatographic purity; water is the pure water, filtered by $0.45 \mu \mathrm{M}$ filter membrane; other reagents are analytical grade.

\subsection{Preparation of Chitosan Microspheres}

Dissolve $0.4 \mathrm{~g}$ chitosan with $20 \mathrm{~mL} 1 \%$ dilute acetic acid, stir and dissolve it fully, then ultrasound till there is no bubble. Take the chitosan solution with a $1 \mathrm{~mL}$ syringe, drop it vertically into $25 \mathrm{~mL}$ of the condensate in a $10 \mathrm{~cm}$ suspension to form chitosan microspheres. After standing for $5 \mathrm{~min}$ at room temperature, washing the microspheres with deionized water to neutral, putting them in $0.1 \%$ glutaraldehyde solution, crosslinking at $30{ }^{\circ} \mathrm{C}$ and 150 rpm for $1 \mathrm{~h}$, washing the crosslinked microspheres with distilled water, the immobilized chitosan carrier is obtained, and stored at $4{ }^{\circ} \mathrm{C}$.

\subsection{Immobilization of $\beta$ - glucosidase bgl2238}

The activity of $\beta$-glucosidase was determined by colorimetry with pNPG as substrate. $\beta$ - galactosidase can catalyze pNPG to produce pNP and glucose. It is yellow in alkaline condition and has absorbance value at $405 \mathrm{~nm}$. The enzyme activity obtained shows the activity of $\beta$ glucosidase completely[31]. Accurately weigh $0.05 \mathrm{~g}$ of immobilized bgl2238, add $20 \mu 150 \mathrm{mM}$ of pNPG, $180 \mu \mathrm{L}$ $\mathrm{pH}$ of 6.10 of B-R buffer solution, mix evenly, after bathing in water at $44{ }^{\circ} \mathrm{C}$ for $20 \mathrm{~min}$, add $400 \mu \mathrm{L}$ of $1 \mathrm{M}$ $\mathrm{Na} 2 \mathrm{CO} 3$ solution to stop the enzymatic reaction, under the same conditions, the inactivated crude enzyme solution is set as the blank control, take $200 \mu \mathrm{L}$ of the above mixture and add it to 96 well plates, measure $\mathrm{OD}_{405}$ in three parallel experiments. An enzyme activity unit (U) is defined as the amount of enzyme required to decompose PNPG in one minute to produce $1 \mu \mathrm{mol} \mathrm{pNP}$.

\subsection{Determination of Residual enzyme activity of immobilized $\beta$ - glucosidase bgl2238}

The total enzyme activity of the immobilized bg12238 was obtained by multiplying the enzyme activity of the immobilized bgl2238 by its total mass. The recovery of enzyme activity is equal to the total enzyme activity of the immobilized bg12238 divided by the enzyme activity of the immobilized crude enzyme solution.

\subsection{Study on the immobilization of bgl2238 on Chitosan Microspheres}

Single factor method was used to optimize the crosslinking conditions (glutaraldehyde concentration, crosslinking time, crosslinking temperature) and the immobilization process (adsorption time and enzyme addition) of $\operatorname{bgl} 2238$.

\subsection{Study on the preparation of immobilized bgl2238 microspheres}

Under the optimum conditions of bgl2238 immobilization, the effects of sodium hydroxide concentration, methanol concentration, acetic acid concentration and chitosan concentration on bgl2238 immobilization were explored.

\subsection{Enzymatic properties of immobilized bgl2238}

\subsubsection{Optimum temperature and temperature stability of immobilized bg/2238}

After adding $0.5 \mathrm{~g}$ immobilized bg12238 to $1.5 \mathrm{~mL}$ EP tube and $20 \mu 150 \mathrm{~mm}$ PNPG, a $200 \mu \mathrm{L}$ enzymolysis system was constructed with B-R buffer of ph6.10. After mixing, the reaction was carried out at different temperatures $\left(35^{\circ} \mathrm{C}\right.$ $-60^{\circ} \mathrm{C}$ ) for 20 minutes, $400 \mu \mathrm{L}$ of $1 \mathrm{~m} \mathrm{Na} 2 \mathrm{CO} 3$ solution was added to stop the enzymatic reaction, $200 \mu \mathrm{L}$ of the reaction solution was added to 96 well plates, and $\mathrm{OD}_{405}$ was determined in three parallel experiments. The maximum enzyme activity was defined as $100 \%$, and the optimum temperature for immobilization of bgl2238 was determined. Then the reaction system of immobilized bgl2238 was placed in $\left(35^{\circ} \mathrm{C}-60{ }^{\circ} \mathrm{C}\right)$ for 1 The residual enzyme activity was measured at the optimum temperature of the immobilized bgl2238. The temperature stability of the immobilized bgl2238 was determined with the enzyme activity of $100 \%$ without heat treatment.

\subsubsection{Optimal $\mathrm{pH}$ and $\mathrm{pH}$ stability of immobilized bg/2238}

The procedure was carried out according to the method 2.7.1 above, keep the optimal temperature unchanged, and determine the optimal $\mathrm{pH}$ value of the immobilized bgl2238 in the $\mathrm{pH}$ range of 3-11. Then the reaction system of immobilized bgl2238 was put into B-R buffer with $\mathrm{pH}$ of 3-11, respectively. After 1 hour at room temperature, the residual enzyme activity of immobilized bg12238 was measured at the optimum temperature. The $\mathrm{pH}$ stability of immobilized bgl2238 was measured with the enzyme 
activity of untreated $100 \%$.

\subsubsection{Effect of metal ions on the immobilization of bg/2238}

$0.5 \mathrm{~g}$ immobilized bgl2238 was put into the solution of $\mathrm{CuSO}_{4}, \mathrm{CoCl}_{2}, \mathrm{FeSO}_{4}, \mathrm{CaCl}_{2}, \mathrm{MgSO}_{4}$ and $\mathrm{MnCl}_{2}$ with the concentration of $10 \mathrm{~mm}$ respectively, and placed for 1 day at room temperature. The activity of the remaining enzyme was measured at the optimum temperature of the immobilized bgl2238. The activity of the enzyme without metal ion treatment was $100 \%$, and the effect of metal ion on the immobilized bgl2238 was measured.

\section{Results and analysis}

\subsection{Bgl2238 immobilization process}

The immobilization process of bgl2238 was studied. The immobilization parameters, such as time, temperature, glutaraldehyde concentration and enzyme dosage, were investigated. The effect of cross-linking time on the activity of immobilized enzyme bgl2238 is shown in Figure $1 \mathrm{~A}$. the specific activity and recovery rate of immobilized enzyme bgl2238 increase in $0.5-1 \mathrm{~h}$, and reach the maximum in $1 \mathrm{~h}$. The best crosslinking time should be the time when the fixed amount of chitosan to bg12238 reaches equilibrium. The crosslinking time is too short, and the enzyme of carrier crosslinking is less. With the prolongation of crosslinking time, the crosslinking reaction is gradually completed, and the enzyme activity is increased. If the crosslinking time is prolonged, the denaturation probability of glutaraldehyde to enzyme will be increased[31].The best immobilization time was $1 \mathrm{~h}$. When the crosslinking temperature rises to $20{ }^{\circ} \mathrm{C}$, the recovery rate and specific enzyme activity of immobilized bgl2238 are the largest (Fig. 1B), but the enzyme activity decreases when the temperature continues to rise. The reason is that when the temperature is too high, the aldehyde group in glutaraldehyde and lactase amino group combine too fast, resulting in the increase of the steric hindrance of enzyme and the decrease of enzyme activity[31]. And the high temperature environment makes the enzyme structure damage, and the enzyme with weak binding with chitosan falls off from the carrier, so the optimal cross-linking temperature is $20^{\circ} \mathrm{C}$.

It is reported that the amount of crosslinking agent will affect the degree of crosslinking. The aldehyde group in glutaraldehyde can covalently combine with the amino group in the enzyme, and also can combine with the active group in the carrier. Therefore, glutaraldehyde can improve the immobilization efficiency of Chitosan on the enzyme[32, 33]. When the concentration of glutaraldehyde is low, the active groups in chitosan are less, which can not form enough cross-linking to fix the enzyme. With the increase of the concentration of glutaraldehyde solution, the carrier is activated, and the amount of enzyme immobilization also increases. At a higher concentration, excessive cross-linking may lead to the denaturation and deactivation of the enzyme structure [34-36]. The effect of glutaraldehyde concentration on the immobilization effect of bgl2238 is shown in Figure 1C. The concentration of glutaraldehyde has a significant impact on the immobilization effect of bgl2238. When the concentration of glutaraldehyde is $0.5 \%$, the immobilization effect of bgl2238 is the best.

In terms of technology, saving the amount of enzyme added is an important factor to be considered, so it is necessary to investigate the amount of enzyme in bg12238[37]. the active groups coupled with enzyme on chitosan microspheres are certain, with the increase of enzyme concentration, these coupling sites will gradually saturate, and the enzyme activity of immobilized enzyme will increase. However, when the enzyme is nearly saturated, a large number of enzyme molecules are gathered in a narrow space, which changes the conformation of the enzyme activity space, but reduces the enzyme activity of the immobilized enzyme. With the increase of enzyme concentration, these coupling sites will gradually saturate and the enzyme activity of immobilized enzyme will increase. However, when the enzyme is nearly saturated, a large number of enzyme molecules are gathered in a narrow space, which changes the conformation of the enzyme activity space, but reduces the enzyme activity of the immobilized enzyme[38]. With the increase of the dilution ratio of bgl2238 crude enzyme solution, the the recovery rate of enzyme activityand specific enzyme activity of the immobilized bg12238 increased first and then decreased. When the dilution ratio of bgl2238 crude enzyme solution was 2 times, the immobilization effect of bgl2238 was the best (Figure 1D). The results of the study on the immobilization process of bgl2238 showed that the optimal crosslinking time was 1 $\mathrm{h}$, the optimal crosslinking temperature was $20^{\circ} \mathrm{C}$, when the concentration of glutaraldehyde was $0.5 \%$, and the dilution of bgl2238 crude enzyme solution was twice, the immobilization effect of $\operatorname{bg} 12238$ was the best.

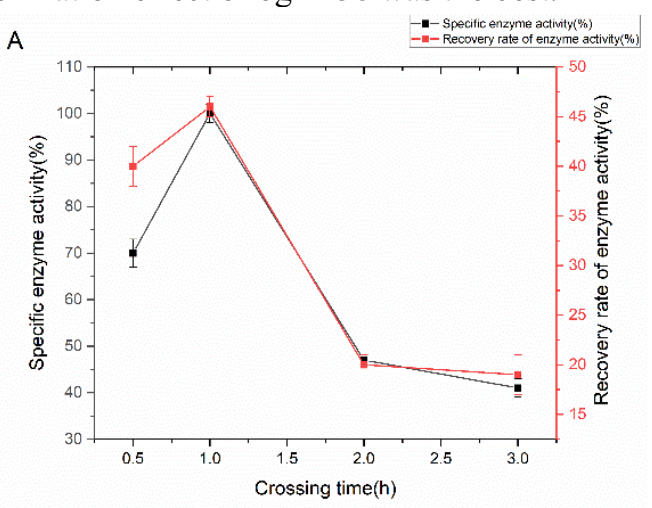


B

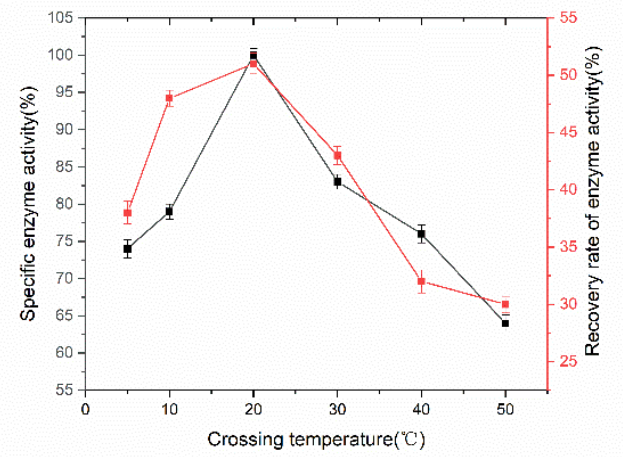

$c$
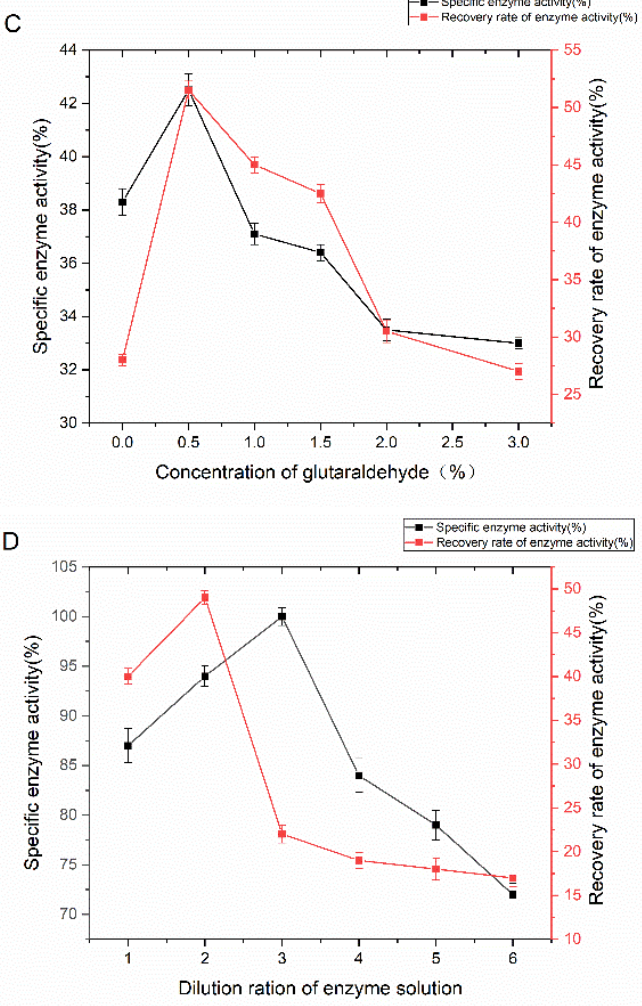

FIg.1 Effects of crosslinking time. temperature. glutaraldehyde concentration and enzyme dosage on the activity of immobilized enzyme bgl2238 (A)Effects of crosslinking time. (B)Effects of crosslinking temperature (C)Effects of glutaraldehyde concentration. (D)Effects of enzyme dosage. Date points are the average of triplicate measurements, and error bars represent the standard deviation

\subsection{Preparation conditions of bgl2238 immobilized on Chitosan}

The effects of the concentration of $\mathrm{NaOH}$, methanol, acetic acid and chitosan on the immobilization of bgl2238 were studied. The concentration of $\mathrm{NaOH}$ can affect the charge of carrier and enzyme, and change the electrostatic attraction between them. A certain concentration of $\mathrm{NaOH}$ can increase the number of deprotonamide, keep the uniformity of the solution but not lead to the precipitation of the polymer. The results showed that with the increase of $\mathrm{NaOH}$ concentration, the activity recovery and specific activity of immobilized bgl2238 increased first and then decreased, but the specific activity was the highest when the concentration of $\mathrm{NaOH}$ was $15 \%$, and the

concentration of $\mathrm{NaOH}$ was $25 \%$ when the activity recovery was the highest. Therefore, according to the proportion of specific enzyme activity and the recovery rate of enzyme activity ratio of $1: 2$, the optimal concentration of $\mathrm{NaOH}$ is $15 \%$ (Figure $2 \mathrm{~A}$ ).

The solvent in the reaction can affect the catalytic performance, because the catalytic performance of the enzyme is closely related to the molecular structure of the enzyme, and methanol causes the conformational change of the enzyme to change the catalytic performance of the immobilized enzyme[39, 40]. At the same time, methanol in the condensate can increase the surface porosity of chitosan microspheres. Although the mechanical strength of the microspheres is weakened, it also increases the probability of enzyme and carrier connection. As shown in Figure $2 \mathrm{~B}$, when the methanol concentration is $20 \%$, the immobilization effect of bgl2238 is the largest. As shown in Figure 2C, when the acetic acid concentration is $1.5 \%$, the recovery rate of enzyme activity and specific activity of bgl2238 immobilized enzyme are the largest.

With the increase of chitosan concentration, on the one hand, it can provide more free aldehydes for covalent connection, on the other hand, the space of carrier becomes smaller, which affects the entry of enzyme and reduces the effective connection between enzyme and carrier, so the concentration of chitosan affects the immobilization effect. As shown in Figure 2D, the optimal concentration of chitosan is $2.0 \%$ when bgl2238 is immobilized. The results showed that when the concentration of $\mathrm{NaOH}$ was $15 \%$, the concentration of methanol was $20 \%$, the concentration of acetic acid was $1.5 \%$, and the concentration of chitosan was $2.0 \%$, the immobilization effect was the best.
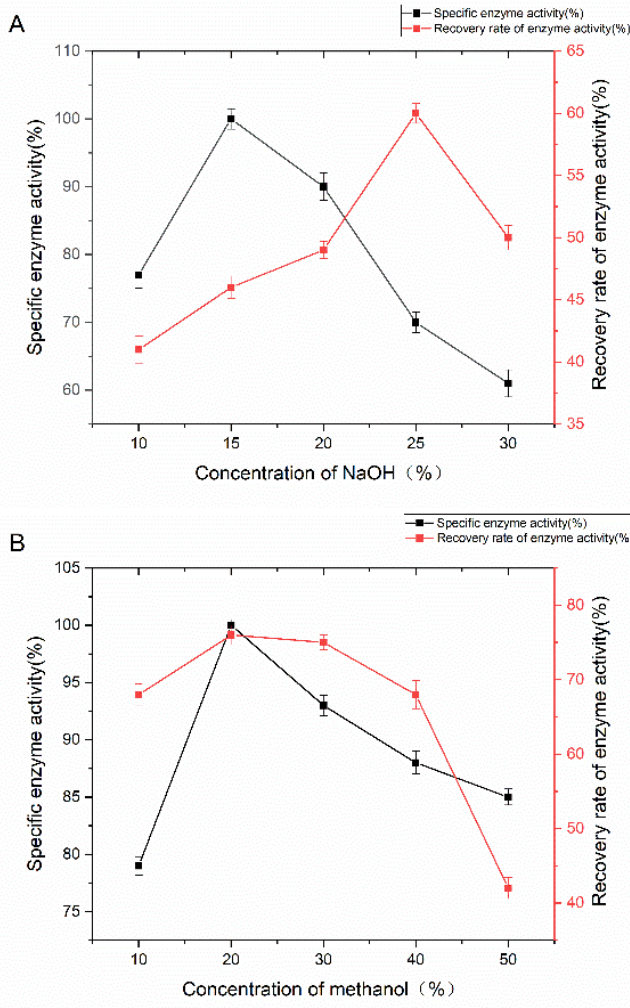

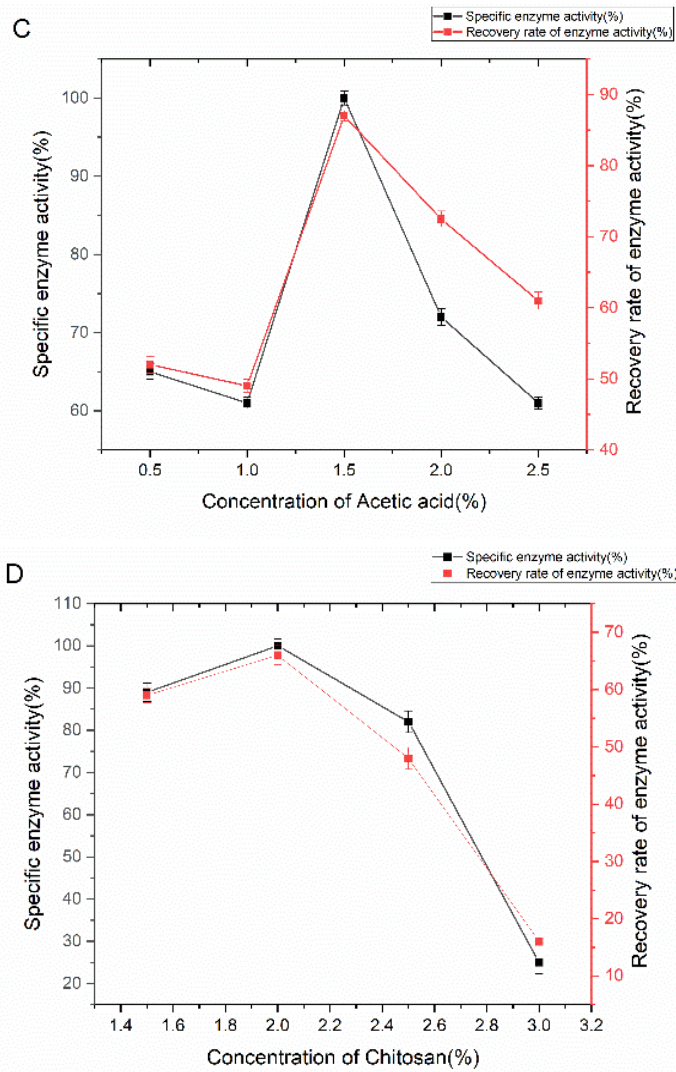

Fig. 2 Effect of $\mathrm{NaOH}$ concentration, methanol concentration, acetic acid concentration and chitosan concentration on the immobilization effect of bgl2238 (A) Effect of $\mathrm{NaOH}$ concentration (B) Effect of methanol concentration (C) Effect of acetic acid concentration (D) Effect of chitosan concentration. Data points are the average of triplicate measurements, and error bars represent the standard deviation

\subsection{Study on the properties of immobilized bgl2238}

\subsubsection{Effect of temperature and $\mathrm{pH}$ on immobilized bg/2238}

In order to determine the optimal temperature of bgl2238, the catalytic activity of the enzyme in the temperature range of $35{ }^{\circ} \mathrm{C}-60{ }^{\circ} \mathrm{C}$ was investigated. The optimal reaction temperature and temperature stability of free bgl2238 and immobilized bgl2238 are shown in Figure A and Figure 3B. It can be seen from Figure 3A that the enzyme activity of immobilized bgl2228 is the largest at $50{ }^{\circ} \mathrm{C}$, while that of free bgl2238 is the largest at $44{ }^{\circ} \mathrm{C}$, and the optimal temperature of immobilized bgl2228 is raised by $6{ }^{\circ} \mathrm{C}$. It can be seen from Figure $\mathrm{B}$ that the free bgl2238 has almost no activity at $50{ }^{\circ} \mathrm{C}$, while the immobilized bgl2238 still has $37 \%$ activity at $50{ }^{\circ} \mathrm{C}$. Therefore, compared with the free bgl2238, the immobilized bgl2238 has a significant improvement in both the temperature range and the temperature stability. It can be seen that the optimal temperature and thermal stability of the immobilized bgl2238 are slightly increased, because the multi-point covalent connection between the immobilized enzyme and the carrier improves the conformational stability of the enzyme molecule[41].
The optimal $\mathrm{pH}$ of bgl2238 is shown in Figure 3C. The optimal $\mathrm{pH}$ after immobilization is still 6.0, which has no change compared with free bgl2238, but the enzyme activity of bgl2238 after immobilization in the range of pH4.0-6.0 has been significantly improved. Figure 3D is the experimental result of the $\mathrm{pH}$ stability of bgl2238. It can be seen from the figure that the stability of the immobilized bgl2238 is much higher than that of the free bgl2238 after being placed for $1 \mathrm{~h}$ at $\mathrm{pH} 4.0-6.0$.
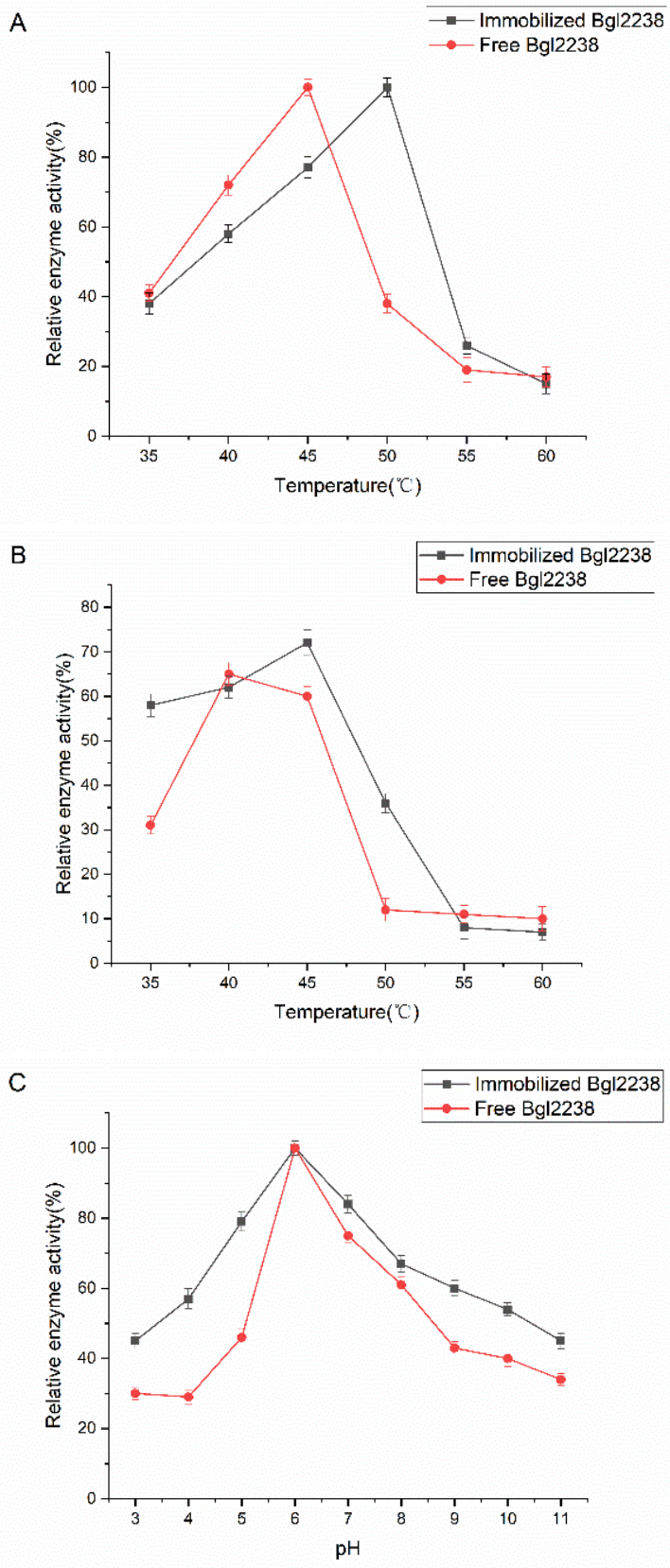


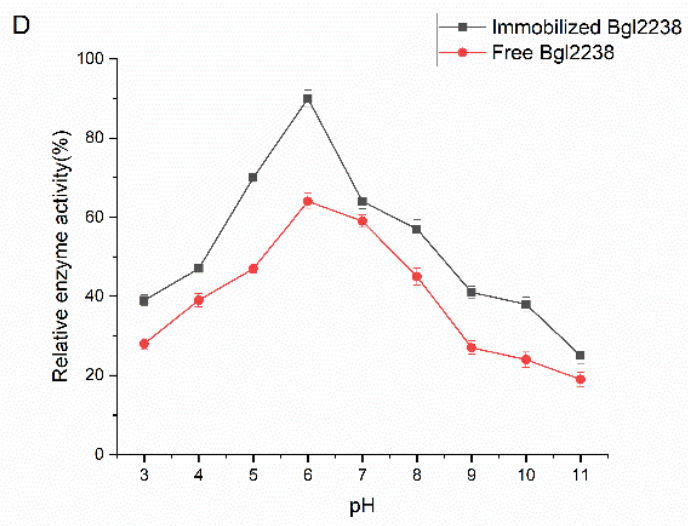

Fig. 3 Effect of $\mathrm{pH}$ and temperature on the activity and stability of free (blue line) and immobilized $\beta$-glucosidase (black line). (A) The optimum temperature of free bg12238 (blue line) and immobilized bgl2238 (black line). (B) Temperature stability of free bgl2238 (blue line) and immobilized bgl2238 (black line) (C) Optimal pH of free bgl2238 (blue line) and immobilized bgl2238 (black line) (D) $\mathrm{pH}$ stability of free bgl2238 (blue line) and immobilized bgl2238 (black line). Data points are the average of triplicate measurements, and error bars represent the standard deviation

\subsubsection{Effect of metal ions on the immobilization of bg/2238}

The effects of metal ions $\mathrm{Cu}^{2+}, \mathrm{Co}^{2+}, \mathrm{Fe}^{2+}, \mathrm{Ca}^{2+}, \mathrm{Mg}^{2+}$ and $\mathrm{Mn}^{2+}$ on the immobilized bgl2238 and free bgl2238 are shown in Figure 4. The figure shows that compared with the free bgl2238, $\mathrm{Ca}^{2+}$ and $\mathrm{Mg}^{2+}$ can enhance the promotion of the immobilized bgl2238, but $\mathrm{Co}^{2+}, \mathrm{Fe}^{2+}$, $\mathrm{Mn}^{2+}$ can weaken the promotion of the immobilized bgl2238, and $\mathrm{Cu}^{2+}$ can also reduce the inhibition of the immobilized bgl2238. This may be because metal ions repel chitosan with positive charge, which reduces the contact between metal ions and enzyme molecules, thus weakening the promotion and inhibition of metal ions on immobilized bgl2238.

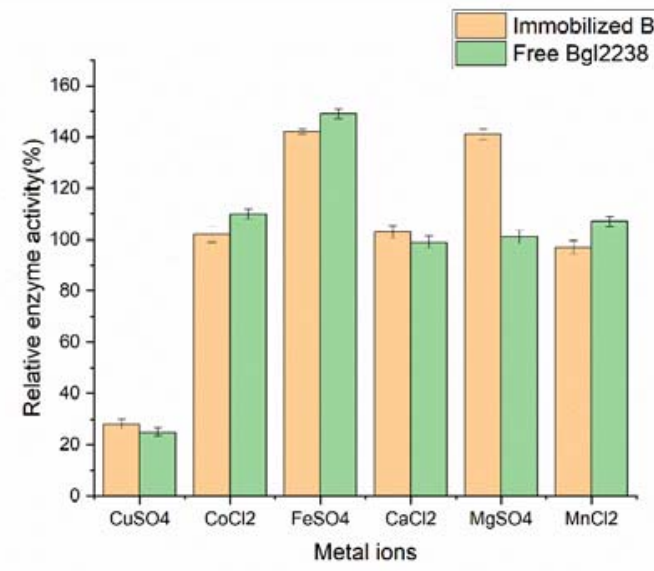

Fig.4 Effect of metal ions on the activity of free bgl2238 and immobilized bg12238.

\section{4 reuse of immobilized bgl2238}

Weigh $12 \mathrm{~g}$ of immobilized bgl2238 to $1 \mathrm{~g}$ of Polygonum cuspidatum powder and hydrolyze it in $10 \mathrm{~mL}$ system at
$50{ }^{\circ} \mathrm{C}$ and $120 \mathrm{rpm}$ for $12 \mathrm{~h}$. The degradation rate of polydatin by free bgl 2238 was $100 \%$, and the degradation rate of polydatin was still over $70 \%$ after the same enzymolysis system was explained for 6 times. (Table 1).

Table. 1 continuous hydrolysis of Polygonum cuspidatum by immobilized bgl2238

\begin{tabular}{cc}
\hline Repetitions & $\begin{array}{c}\text { Degradation rate of } \\
\text { polydatin }(\%)\end{array}$ \\
\hline 1 & 95 \\
2 & 89 \\
3 & 83 \\
4 & 80 \\
5 & 76 \\
6 & 72 \\
\hline
\end{tabular}

\section{Conclusions}

$\beta$ - glucosidase bgl2238 has significant degradation effect on polydatin. Immobilization of bgl2238 can not only reuse, but also improve the stability of the enzyme. Operational stability or reusability is an important index to evaluate the efficiency of immobilized enzyme[37]. After hydrolysis of Polygonum cuspidatum for six times, the degradation rate of polydatin is still over $70 \%$, which proves that the immobilized bgl2238 has good reusability. Temperature stability and $\mathrm{pH}$ stability were improved. The results show that the immobilization process is simple, the carrier material is easy to obtain, and the enzyme activity and stability are improved, which shows the effectiveness and feasibility of the process and determines the best conditions for its production[42]. It also laid a foundation for the application of immobilized $\beta$ - glucosidase in practice.

\section{Funding}

This work was supported by Natural Science Foundation of China (31400680), Science and Technology Plan Project of Guangzhou (201802030009), Innovation and Strengthening School Project from Guangdong Pharmaceutical University (2016SFKC_28 and 2016KTSCX067), Science and Technology Plan Project of Guangdong Province (2017 A010105011), Science and Technology Plan Project of Guangzhou (201802030009). National Natural Science Foundation of China (31971384).

\section{References}

1. Carrizzo, A., et al., Antioxidant effects of resveratrol in cardiovascular, cerebral and metabolic diseases. Food \& Chemical Toxicology, 2013. 61: p. 215-226.

2. Alkhalaf and Moussa, Resveratrol-induced growth inhibition in MDA-MB-231 breast cancer cells is associated with mitogen-activated protein kinase signaling and protein translation. European Journal of Cancer Prevention, 2007. 16(4): p. 334-341.

3. Atten, M.J., et al., Resveratrol regulates cellular PKC $\alpha$ and $\delta$ to inhibit growth and induce apoptosis in 
gastric cancer cells. Investigational New Drugs, 2005. 23(2): p. 111-119.

4. Castino, N.F.T.G.N.C.F.R. and C. Isidoro, Resveratrol induces cell death in colorectal cancer cells by a novel pathway involving lysosomal cathepsin D. Carcinogenesis, 2007. 28(5): p. 922-931.

5. Holian, O., Resveratrol regulates cellular PKC $\alpha$ and $\delta$ to inhibit growth and induce apoptosis in gastric cancer cells. Investigational New Drugs. 23(2): p. 111-119.

6. Holian, O., Resveratrol regulates cellular PKC $\alpha$ and $\delta$ to inhibit growth and induce apoptosis in gastric cancer cells. Investigational New Drugs, 2005. 23(2): p. 111-119.

7. Baile, C.A., et al., Effect of resveratrol on fat mobilization. Annals of the New York Academy of Sciences, 2011. 1215: p. p.40-47.

8. Wong, R.H.X., et al., Chronic resveratrol consumption improves brachial flow-mediated dilatation in healthy obese adults. Journal of Hypertension, 2013. 31(9): p. 1819-1827.

9. Rivera, L., et al., Long-term resveratrol administration reduces metabolic disturbances and lowers blood pressure in obese Zucker rats. 2009. 77(6): p. 1053-1063.

10. Szkudelski, T. and K. Szkudelska, Resveratrol and diabetes: from animal to human studies. Biochimica Et Biophysica Acta, 2015. 1852(6): p. 1145-1154.

11. Palsamy, P. and S. Subramanian, Resveratrol, a natural phytoalexin, normalizes hyperglycemia in streptozotocin-nicotinamide induced experimental diabetic rats. Biomedicine \& Pharmacotherapy, 2008. 62(9): p. 0-605.

12. Pasinetti, G.M., et al., Neuroprotective and metabolic effects of resveratrol: Therapeutic implications for Huntington $\mid$ "s disease and other neurodegenerative disorders. 2011. 232(1): p. 1-6.

13. Berman, A.Y., et al., The therapeutic potential of resveratrol: a review of clinical trials. Npj Precision Oncology, 2017. 1(1): p. 35.

14. Zhang, F., J. Liu, and J.S. Shi, Anti-inflammatory activities of resveratrol in the brain: Role of resveratrol in microglial activation. European Journal of Pharmacology, 2010. 636(1-3): p. 1-7.

15. Bradamante, S., L. Barenghi, and A. Villa, Cardiovascular Protective Effects of Resveratrol. Cardiovascular Therapeutics, 2004. 22(3): p. 169-188.

16. Samarjit Das, D.K.D., Resveratrol: A Therapeutic Promise for Cardiovascular Diseases. Recent Patents on Cardiovascular Drug Discovery, 2007. 2(2): p. p.133-138.

17. Baur, J.A. and D.A. Sinclair, Therapeutic potential of resveratrol: the in vivo evidence. Nature Reviews Drug Discovery, 2006. 5(6): p. 493-506.

18. Vestergaard, M. and H. Ingmer, Antibacterial and Antifungal Properties of Resveratrol. International Journal of Antimicrobial Agents.
19. T, R., et al., Preventive role of Resveratrol against inflammatory cytokines and related diseases. Curr Pharm Des., 2019.

20. Singh, A.P., et al., Health benefits of resveratrol: Evidence from clinical studies. Medicinal Research Reviews, 2019.

21. Zhang, Y., et al., Insight into the assembly of rootassociated microbiome in the medicinal plant Polygonum cuspidatum. Industrial Crops and Products, 2020. 145.

22. Wang, C., et al., Efficient Enzyme-Assisted Extraction and Conversion of Polydatin to Resveratrol From Polygonum cuspidatum Using Thermostable Cellulase and Immobilized $\beta$-Glucosidase. Frontiers in Microbiology, 2019.

23. Uhoraningoga, A., et al., The Statistical Optimisation of Recombinant beta-glucosidase Production through a Two-Stage, Multi-Model, Design of Experiments Approach. Bioengineering (Basel), 2019. 6(3).

24. Zhang, D.-Y., et al., Fabrication of three-dimensional porous cellulose microsphere bioreactor for biotransformation of polydatin to resveratrol from Polygonum cuspidatum Siebold \& Zucc. Industrial Crops and Products, 2020. 144.

25. Guisan, J.M., et al., The Science of Enzyme Immobilization. Methods Mol Biol, 2020. 2100: p. 126.

26. Bilal, M., et al., Multi-point enzyme immobilization, surface chemistry, and novel platforms: a paradigm shift in biocatalyst design. Critical Reviews in Biotechnology, 2019.

27. Sannino, F., et al., Covalent Immobilization of betaGlucosidase into Mesoporous Silica Nanoparticles from Anhydrous Acetone Enhances Its Catalytic Performance. Nanomaterials (Basel), 2020. 10(1).

28. Tu, M., et al., Immobilization of $\beta$-glucosidase on Eupergit C for Lignocellulose Hydrolysis. 2006. 28(3): p. 151-156.

29. Yue, W., et al., In situ preparation of magnetic Fe3O4-chitosan nanoparticles for lipase immobilization by cross-linking and oxidation in aqueous solution. 2009. 100(14): p. 3459-3464.

30. Bilal, M. and H.M.N. Iqbal, Naturally-derived biopolymers: Potential platforms for enzyme immobilization. Int J Biol Macromol, 2019. 130: p. 462-482.

31. Chen, H., et al., The Effect of Glutaraldehyde CrossLinking on the Enzyme Activity of Immobilized \&betaGalactosidase on Chitosan Bead. Advance Journal of Food Science and Technology, 2013. 5(7): p. 932-935.

32. Adriano, W.S., et al., Stabilization of penicillin $G$ acylase by immobilization on glutaraldehydeactivated chitosan. Brazilian Journal of Chemical Engineering, 2005. 22: p. 529-538.

33. Migneault, I., et al., Glutaraldehyde: Behavior in aqueous solution, reaction with proteins, and application to enzyme crosslinking. 2004. 37(5): p. 798-802. 
34. Chui, W.K. and L.S.C. Wan, Prolonged retention of cross-linked trypsin in calcium alginate microspheres. Journal of Microencapsulation, 1997. 14(1): p. 51-61.

35. Broun, G.B., [20] Chemically aggregated enzymes. Methods in Enzymology, 1976. 44: p. 263-280.

36. Lopez-Gallego, F., et al., Enzyme stabilization by glutaraldehyde crosslinking of adsorbed proteins on aminated supports. J Biotechnol, 2005. 119(1): p. 705.

37. DiCosimo, R., et al., Industrial use of immobilized enzymes. Chem Soc Rev, 2013. 42(15): p. 6437-74.

38. Jiang, D.S., et al., Immobilization of Pycnoporus sanguineus laccase on magnetic chitosan microspheres. Biochemical Engineering Journal, 2005. 25(1): p. 15-23.

39. Fernández-Lafuente, R., C.M. Rosell, and J.M. Guisán, The presence of methanol exerts a strong and complex modulation of the synthesis of different antibiotics by immobilized Penicillin $G$ acylase. Enzyme \& Microbial Technology, 1998. 23(5): p. 305-310.

40. Kumari, A., et al., Enzymatic transesterification of Jatropha oil. Biotechnology for Biofuels, 2009. 2(1): p. 1.

41. Park, T.G. and A.S. Hoffman, Immobilization of Arthrobacter simplex in a thermally reversible hydrogel: Effect of temperature cycling on steroid conversion. Biotechnology \& Bioengineering, 1990. 35(2): p. 152-159.

42. Gianfreda, L. and J.-M. Bollag, Effect of Soils on the Behavior of Immobilized Enzymes. Soil Science Society of America Journal, 1994. 58(6): p. 1672. 